Original Paper http://ajol.info/index.php/ijbcs http://indexmedicus.afro.who.int

\title{
Etude socio-économique de la pêche dans la partie Sud-Ouest du lac Albert (Ituri, RD Congo)
}

\author{
Cikwanine KASIGWA ${ }^{1 *}$, Joseph MATUNGURU², Nshombo MUDERHWA ${ }^{3,}$ \\ Jonas JARIEKONGA ${ }^{4}$, Alidor KANKONDA ${ }^{5}$ et Jean-Claude MICHA ${ }^{6}$ \\ ${ }^{1}$ Université Officielle de Bukavu, Faculté des sciences, Département de Biologie, Bukavu, Sud-Kivu, RDC. \\ ${ }^{2}$ Ecole Doctorale de l'Université du Burundi, Burundi. \\ ${ }^{3}$ Centre de Recherche en Hydrobiologie, Uvira, Sud-Kivu, RDC. \\ ${ }^{4}$ Lakes Edward and Albert Fishiries Project, Bunia, Ituri, RDC. \\ ${ }^{5}$ Université de Kisangani, Faculté des sciences, Département d'Hydrobiologie, Kisangani, RDC \\ ${ }^{6}$ Environmental Biology Research Unit (URBE), University of Namur, Namur, Belgium. \\ *Auteur correspondant ; E-mail : cikwadieudonne@gmail.com
}

\section{RESUME}

Une étude socio-économique a été conduite sur 602 pêcheurs répartis dans 16 camps de pêche dans la partie sud-ouest du lac Albert dans le but d'évaluer les activités des pêcheries. Dans chaque camp, les engins de pêche ont été identifiés, caractérisés et dénombrés ; et la composition des captures de poisson évaluée par type d'engin. 8 engins de pêche ont été identifiés, y compris des engins prohibés selon la réglementation de 2016, notamment le filet monofilament et les nasses. La palangre $(1343,64 \pm 832,5 \mathrm{~m})$, le filet dormant de fond $(1053,05$ $\pm 520 \mathrm{~m})$ et le filet dormant de surface $(766,26 \pm 536 \mathrm{~m})$ ont été les plus utilisés. La senne de tournante était identifiée comme l'engin le plus productif, avec 183,18 \pm 49,02 kg/jour pendant la bonne période et 20,89 \pm $12,94 \mathrm{~kg} /$ jour pendant la mauvaise période), mais elle a été aussi la moins sélective de tous les engins identifiés. La pêche au filet dormant a donné une production journalière d'environ 62,7 kg en moyenne pendant la bonne période et de $10 \mathrm{~kg}$ pendant la mauvaise. L'occupation des zones de frayère, l'augmentation de l'effort de pêche, et l'utilisation des engins prohibés étaient considérées comme causes majeures de la baisse de productivité du lac Albert. Il demeure urgent d'appliquer la réglementation afin de protéger l'ichtyofaune du lac.

(C) 2020 International Formulae Group. All rights reserved.

Mots clés : Organisation, pêcheries, Sud-Ouest, lac Albert.

\section{Socio-economic study of fisheries in the southwest part of Lake Albert (Ituri, DR Congo)}

\begin{abstract}
A socio-economic study was conducted on 602 fishermen in 16 fishing camps in the southwestern part of Lake Albert in order to assess the activities of the fisheries. In each camp, the fishing gear was identified, characterized and counted; and the composition of fish catches assessed by gear type. 8 fishing gear have been identified, including gear prohibited under the 2016 regulations, notably the monofilament net and the nets. The length $(1343.64 \pm 832.5 \mathrm{~m})$, the bottom fixed net $(1053.05 \pm 520 \mathrm{~m})$ and the surface fixed net $(766.26 \pm 536 \mathrm{~m})$ were the most used. The purse seine was identified as the most productive gear, with $183.18 \pm 49.02 \mathrm{~kg} /$ day
\end{abstract}


during the good period and $20.89 \pm 12.94 \mathrm{~kg}$ / day during the bad period), but it was also the least selective of all the gear identified. The dormant net fishery gave an average daily production of around $62.7 \mathrm{~kg}$ during the good period and $10 \mathrm{~kg}$ during the bad period. The occupation of the spawning grounds, the increase in fishing effort, and the use of prohibited gear were considered as major causes of the decline in productivity of Lake Albert. There remains an urgent need to enforce regulations to protect the lake's fish fauna.

(C) 2020 International Formulae Group. All rights reserved.

Keywords: Organization, fisheries, Southwest, Lake Albert.

\section{INTRODUCTION}

Depuis les temps les plus reculés, les océans, les lacs et les cours d'eau sont une source importante d'aliments, la pêche assurant des emplois et d'autres avantages économiques à l'humanité (FAO, 2018) et procure plus 70\% de protéines à la population africaine (Micha et Franck, 2004 ; Ticheler, 2000 ; Idowu, 2010). Les ressources des océans en particulier semblaient illimitées. Toutefois, avec l'enrichissement des connaissances et le développement dynamique du secteur des pêches, l'humanité commence à comprendre que les ressources halieutiques, certes renouvelables, ne sont pas infinies et doivent être gérées à bon escient si l'on veut maintenir leur contribution au bien-être nutritionnel, économique et social de la population croissante de la planète ; (FAO, 2010). Selon les statistiques de la FAO (2008), la pêche artisanale fait vivre plus de 100 millions de personnes dans le monde, mais la plupart des petits pêcheurs s'appauvrissent d'années en années et $95 \%$ des captures continentales proviennent des pays en voie de développement dont $90 \%$ de cette production sont consommés par eux-mêmes (Bartley et al., 2015).

Le problème de fond d'un secteur fondé sur l'exploitation des ressources naturelles est la surexploitation d'un stock de ressources épuisables (Burac, 2006). La surpêche ne se mesure pas uniquement au grand nombre de pêcheurs par unité de surface, mais aussi à l'augmentation du nombre et de la taille des engins qui peuvent modifier l'écosystème aquatique (Paugy et Lévêque, 2017). A cet effet, Chauveau (2000) signale que la croissance démographique rapide et massive des populations riveraines, la diffusion de techniques de pêche plus performantes (moteurs et engins de pêche souvent peu sélectifs comme la senne tournante) se sont traduites par une pression accrue sur le capital halieutique alors que les conditions de l'environnement se détériorent. Or, ces peuplements de poissons sont composés des nombreuses espèces parmi lesquelles certaines sont plus fragiles et vulnérables que d'autres et qui résistent moins à la dégradation de leur milieu de vie (Badahoui, 2010). Les équilibres deviennent d'autant plus instables que les efforts de pêche augmentent et que les techniques de pêche se perfectionnent sans tenir compte des ressources disponibles et de la dynamique de population en place (Lévêque et Paugy, $1999 \mathrm{~b} ; 1$ 1999c). Il s'ajoute aux problèmes précédemment cités, la pêche illicite, non déclarée et non réglementée (INN) comme source de destruction d'un écosystème aquatique, (Bergh et Sowman, 2016).

La pêche continentale occupe une place de choix en Afrique avec 1,5 à 2 millions de tonnes par an (Laë et Léveque, 1999). En particulier le poisson fait partie des ressources importantes dans la province d'Ituri située à l'est de la RD Congo et contribue aux revenus de plusieurs milliers de ménages. La source principale de production du poisson dans ce milieu est le lac Albert. Depuis quelques années, on observe une baisse des captures, un prélèvement des spécimens de poissons de petite taille (immatures) suite aux mauvaises pratiques conduisant à la raréfaction, dans les captures, de certaines espèces jadis importantes. Ceci nécessite l'élucidation des causes à la base de cette situation si on veut rendre cette ressource durable.

Pour cela, il s'avère donc urgent d'actualiser les données en rapport avec l'organisation de la pêche, les engins utilisés, 
les espèces et quantités pêchées, les moyens investis par les pêcheurs, le profit réalisé ainsi que les différents défis auxquels ils font face.

\section{MATERIEL ET METHODES \\ Milieu d'étude}

Situé entre $1^{\circ}$ et $2^{\circ} 19^{\prime}$ de latitude Nord et entre $30^{\circ} 33^{\prime}$ et $31^{\circ} 26^{\prime}$ de longitude Est, à 619 mètres d'altitude. Le lac Albert, avec 161 $\mathrm{km}$ de long, $32 \mathrm{~km}$ de large et une superficie de $5.374 \mathrm{~km}^{2}$, est l'un des grands lacs du rift albertin (Messen 1951; Baker, 1869). Deuxième lac en RD Congo sur le plan de superficie, il est influencé par le climat tropical avec des précipitations de l'ordre 1200 à 1800 mm par an. La Figure 1 montre le lac Albert et présente sa partie sud-ouest avec différents camps de pêche.

L'ichtyofaune du lac Albert est relativement pauvre, elle compte 46 espèces réparties dans 18 familles (Fermon, 2007). Le lac Albert présente des biotopes peu diversifiés car il est formé sur des grabens rocheux.

\section{Méthodes de collecte des données}

$\mathrm{Au}$ total 205 patrons pêcheurs et 397 pêcheurs ouvriers ont été questionnés dans 16 camps de pêche. Dans chaque camp, le nombre de pêcheurs, d'engins de pêche y compris leurs caractéristiques (types d'engins, longueur, largeur, maille des engins) étaient enregistrés. D'autres informations notamment celles sur les captures en fonction des techniques de pêche ont été obtenues à l'aide d'un questionnaire d'enquête soumis d'une part aux patrons pêcheurs et d'autre part aux pêcheurs ouvriers. Les interviews et focus groupes ont été réalisés avec les chefs des champs et les agents impliqués dans la gestion de l'écosystème du lac dans la partie d'Irumu.

\section{Traitement et analyse des données}

Le questionnaire d'enquête a été conçu avec le logiciel SPHINX 4.5. Après encodage des réponses, les informations ont été traitées, une partie dans le même logiciel et une autre dans le logiciel SPSS 20 après transfert des données. L'analyse descriptive a été faite pour les données socio-démographiques (tribus, âge), la répartition de tributs, l'âge de pêcheurs, un regroupement des réponses en termes de fréquence et de pourcentage afin de faire une analyse comparative (nombre de membre d'équipage, longueur de différents filets, de barques, nombre de sorties par semaine, le temps de capture et le revenu par engin de pêche). Une corrélation de Pearson a été établie entre afin de vérifier le lien entre la capture en $\mathrm{kg}$ et le prix par $\mathrm{kg}$ en tenant compte de la période. Le test de Chi2 a permis de comparer la répartition les facteurs explicatifs de la diminution des captures, les causes et les pistes de solution.

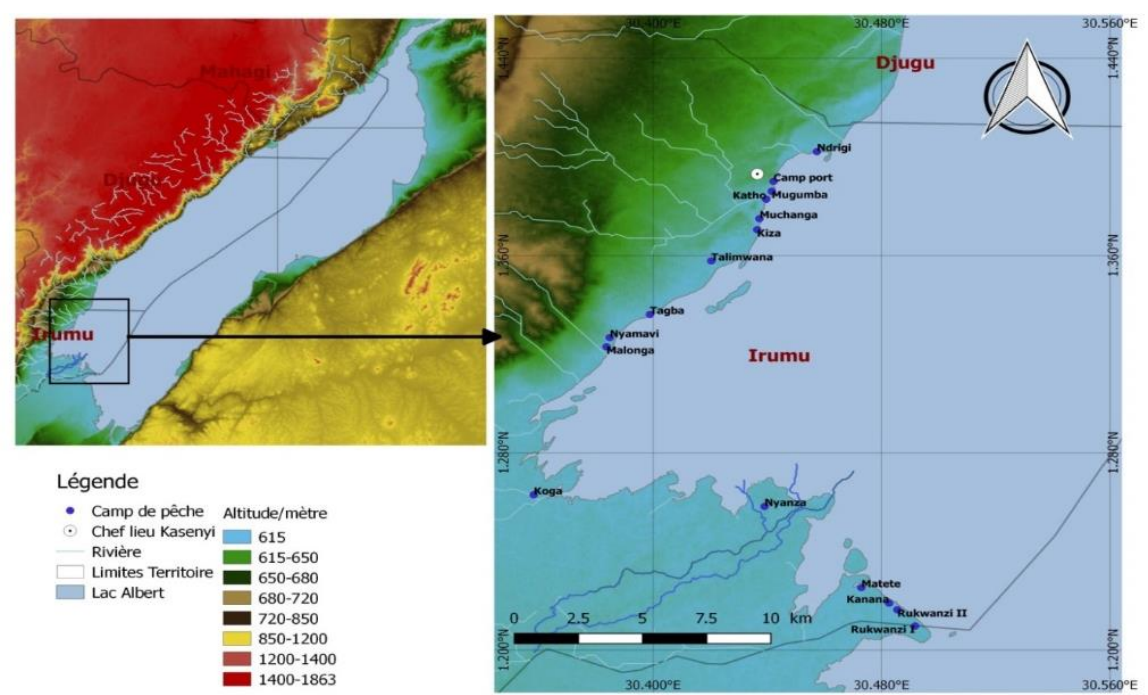

Figure 1 : Position géographique des camps de pêche au Sud-Ouest de la partie RDC du lac Albert. 


\section{RESULTATS}

\section{Résultats socio-démographiques}

Sur 19 tribus identifiées dans 16 camps enquêtés, les Alur occupent la première place avec $34,7 \%$ suivi des Ngiti $(14,2 \%)$ et des Hema (13,9\%). Les Alurs sont nombreux dans l'activité de pêche probablement parce qu'étant établis plus proche du lac (Tableau 1). En effet, la plus grande partie facilement accessible au lac, du côté congolais, se trouve dans leur territoire d'origine, notamment le territoire de Mahagi. Les autres tribus voisines du lac sont les Hema qui sont connus généralement comme éleveurs et les Ngiti qui s'occupent généralement de l'agriculture. Parmi les tribus non originaires de l'Ituri, les Mungelema $(7,6 \%)$ et les Nande $(6,3 \%)$ sont les mieux représentés.

L'âge des pêcheurs (Tableau 2) est de $34,5 \pm 11,16$ ans en moyenne et il se remarque une différence significative entre les moyennes d'âge en fonction des différents camps de pêche $(\mathrm{F}=3,15 ; 1-\mathrm{p}=99,99 \%$ au risque de 95\%). Ainsi c'est dans le camp de pêche Katho que la moyenne d'âge des pêcheurs est la plus élevée (soit 46,3 ans) alors que les pêcheurs les plus jeunes sont dans les camps de pêche Mugumba $(29,1 \pm 9,97$ ans) et Rukwanzi I $(29,9 \pm 8,85)$.

Nos enquêtes révèlent qu'en moyenne $12,7 \%$ de femmes seulement sont armateurs et que les pêcheurs ouvriers sont généralement de sexe masculin. En ce qui concerne le niveau d'instruction, $46,9 \%$ en moyenne de pêcheurs de CO (Cycle d'orientation) suivi de niveau primaire (33,35\% en moyenne). En distinguant les pêcheurs ouvriers des armateurs, il ressort que chez les armateurs 51,3\% sont de $\mathrm{CO}$, $27,3 \%$ de primaire, $14,6 \%$ diplômés et seulement $6,3 \%$ d'analphabètes contre $42,5 \%$ de $\mathrm{CO}, 39,4 \%$ de primaires, $12,6 \%$ d'analphabètes et $3,4 \%$ de diplômés. Ce qui laisse croire que les armateurs sont plus au moins instruits que les pêcheurs ouvriers.

\section{Répartition des armateurs en fonction des camps de pêche}

$\mathrm{Au}$ total 822 armateurs ont été inventoriés avec une moyenne de 51,37 $\pm 20,43$ par camp de pêche. Selon la Figure 2 les camps de pêche les plus pourvus en armateurs sont Koga (100 armateurs) suivie de Rukwanzi I (75 armateurs) alors que l'effectif faible est constaté dans le camp Kanana (32 armateurs). Il sied de dire que le nombre d'armateurs varie beaucoup en fonction au cours de l'année selon les conditions sécuritaires, la fermeture de la pêche, le niveau d'eau, les vagues.

\section{Résultats sur les statistiques des engins de pêche}

Nos enquêtes révèlent que 8 types d'engins de pêche sont utilisés dans la partie sud-ouest du lac Albert. Le Tableau 3 ci-après montre que le filet dormant de surface est le plus utilisé $(29,72 \%)$ suivi du filet dormant de fond $(23,07 \%)$, de monofilament $(13,8 \%)$ et la palangre $(11,18 \%)$. La senne tournante ne représente que $10,54 \%$ et le filet moustiquaire est moins utilisé $(1,34 \%)$ suivi d'épervier $(4,31 \%)$.

En tenant compte de répartition par camp de pêche, il sied de constaté que la senne tournante est plus employée par les pêcheurs de Matete. Le filet moustiquaire/Kituba utilisé lors de la recherche des appâts pour la pêche aux palangres est beaucoup plus employé dans les camps Nyamavi, Nyanzi et Koga.

Les filets maillants sardine (filet de surface qui vise généralement les genres Hydrocynus et Alestes entre 0 et 26 mètres de profondeur) sont plus utilisés dans les camps Koga, Nyanza et Rukwanzi I et guba (filet maillant de fond dont la chute va jusqu'entre 52 et 78 mètres de profondeurs) qui vise le genre Lates. est plus utilisé dans les camps Koga, Nyamavi et Lako. Le filet maillant monofilament accompagné de la frappe de l'eau avec un tronc d'arbre appelé 'tambour' est beaucoup employé dans les camps de pêche Tagba, Koga et Kiza en visant plus la pêche des poissons de la famille de Cichlidae. Les nasses exigeant les eaux moins profondes avec une grande présence de macrophytes sont une spécialité des pêcheurs de Koga, Tagba, Matete et Nyamavi tous situés dans la zone du delta de la Semliki.

Au total 924 barques de pêche ont été inventoriées dans 16 camps de pêche avec une moyenne de 57,75 par camp et un écart-type de 
44,19. Ceci montre, comme renseigne la Figure 3 , que la distribution est inégale et que c'est à Tagba qu'on a un grand nombre de barques $(21,4 \%)$ suivi de Rukwanzi I $(11,6 \%)$ et Matete $(9,3 \%)$. Pour faciliter le déplacement, les pêcheurs utilisent de fois les moteurs. Il a été identifié 63 moteurs dans cette partie du lac et $25,4 \%$ de ces derniers se trouvent concentrés à Tagba suivis de Mugumba (15,87\%) et Camp port $(14,29 \%)$. Notons qu'il existe des camps de pêche où les moteurs ne sont pas utilisés dans les activités de pêche (Camps Nyanza, Kanana, Koga, Nyamavi, Kiza et Lako) comme le montre la même figure. Il est à retenir que plusieurs unités de pêches peuvent utiliser un seul moteur afin d'atteindre les sites de pêche ou évacuer les produits de pêche. Le moteur utilisé est de $15 \mathrm{CV}$ généralement.

\section{Unité de pêche, effort de capture par unité de pêche et caractéristiques des engins de la pêche}

A la lumière du Tableau 4, la pêche dans la partie sud-ouest du lac Albert est essentiellement basée sur trois types principaux engins de pêche (senne, filet maillant et la palangre). En ce qui concerne la senne tournante, une unité de pêche est constituée en moyenne de 10,47 $\pm 2,65$ personnes alors que les autres engins de pêche utilisent en moyenne 1 ou 2 personnes par unité de pêche $(\mathrm{F}=271,46$ et 1-p $=>99,99 \%)$. Quant au nombre de barque par unité de pêche une différence significative se dégage entre la pêche à la senne tournant (1 ou 2 barques selon le cas) contre un seul pour les autres techniques de pêche $(\mathrm{F}=6,58 ; 1$-p => 99,99\%). Il est à signaler que la barque des pêcheurs employant la senne est plus longue (7,41 $\pm 4,4$ mètres en moyenne) que les autres ayant une longueur autour de 4 mètres de long $(\mathrm{F}=16,79 ; 1-\mathrm{p} \Rightarrow 99,99 \%)$. Notons par ailleurs que le nombre de personnes par unité de pêche joue directement sur la grandeur de la barque.

Les filets de pêche différent significativement en longueur $(\mathrm{F}=5,02 ; 1-\mathrm{p}$ $=>99,99 \%$ ) et c'est la palangre est qui la plus longue pouvant atteindre $1343,67 \pm 832,5$ mètres en moyenne suivis du filet dormant de fond $(1053,05 \pm 520$ mètres $)$ et celui de surface (766,26 \pm 536 mètres en moyenne).

Touchant de nombre de sortie de pêche par semaine, il ressort que 5,44 jours est la moyenne hebdomadaire sans distinguer les engins de pêche. Par comparaison, la pêche à la nasse se distingue des autres $(\mathrm{F}=2,42 ; 1-\mathrm{p}=$ $98,51 \%)$ et s'effectue chaque jour $(6,81 \pm 0,87$ en moyenne).

Lors d'une sortie de pêche, un filet selon qu'il est actif ou passif, peut être jeté une ou plusieurs fois. Les filets dormants sont généralement placés une seule fois au cours d'une journée de pêche à l'exception de monofilament $(9,88 \pm 12,32$ fois $)$ alors que ceux actifs sont jetés plusieurs fois $(\mathrm{F}=9,51$; $1-p=>99,99 \%)$. Pour toutes les techniques de pêche, les activités commencent vers 3 à 5 heures du matin ou au coucher du soleil pour les unités de pêche qui passent la nuit sur le lac en train de pêcher. 10,5 heures de pêche sont accomplies généralement pendant un cycle de 24 heures et avec une moyenne de 5,6 sorties au cours d'une semaine. Donc, une unité d'effort de pêche réalise en moyenne 58,8 heures de travail la semaine et 235,2 heures par mois.

Selon les résultats d'interview, les activités de pêche sont plus élevées au mois d'avril suivi de novembre et août selon les pêcheurs. On constate un pic d'activités de pêche et une augmentation des captures aux mois de mars, avril, août et novembre et les captures deviennent sporadiques le reste de l'année. L'analyse du Tableau 5 montre que l'augmentation des activités de pêche correspond à la saison des pluies $(62,1 \%$ des enquêtés) avec une montée de niveau de l'eau (25,9\% des enquêtés) et beaucoup de mouvements de vagues sur le lac $(47,2 \%)$ bien que les pourcentages varient d'un engin de pêche à l'autre. La bonne période correspond à la saison des pluies qui est malheureusement accompagnée de vagues où l'on constate beaucoup de camp de noyade des pêcheurs.

Il y a lieu de signifier que la plupart des unités de pêche mènent leurs activités sur l'ensemble du lac. Le Tableau 6 montre que les utilisateurs de senne se rendent plus dans les zones de grandes profondeurs tandis que ceux 
employant les filets dormants de surface et de fond se trouvent dans les zones littorales, de frayères et riches en macrophytes. Cela est vrai pour le filet dormant monofilament, accompagné de la frappe, considéré comme prohibé au lac Albert.

La détermination de l'espèce cible lors de la pêche est fonction du site et de l'engin de pêche. Il ressort de cette étude que la senne tournante capture une large gamme d'espèces au lac Albert et est moins sélective que les autres engins de pêche. Les genres Bagrus, Lates, Synodontis, Coptodon, Oreochromis, Sarotherodon et Brycinus représentent 20 à $79,4 \%$ des captures en utilisant la senne tournante. Le filet dormant de surface cible plus les genres Hydrocynus, Alestes et quelques individus de Lates et Tilapia tandis que le filet dormant de fond est plus spécifique pour la pêche du genre Lates (plus de 50\%) bien qu'il capture aussi le genre Hydrocynus et les Cichlidae en général. La nasse reste très sélective et capture presque seulement les Cichlidae. La palangre capture plus les genres Bagrus et Clarias. Il se dégage donc généralement sans distinction des engins de pêche que les espèces cibles appartiennent prioritairement aux familles Latiidae, Cichlidae et Alestidae (Tableau 7).

Les captures fluctuent beaucoup en fonction des techniques de pêche, des périodes et des facteurs environnementaux. En analysant le Tableau 8 , on constate pour la senne tournante pendant la bonne période que les captures fluctuent autour de 183,18 \pm 49,02 $\mathrm{kg}$ et se réduisent à $20,89 \pm 12,94 \mathrm{~kg}$ lors de la mauvaise période. La pêche aux filets dormants de surface et de fond donne les résultats quasi-identiques pendant les deux périodes (respectivement $62,7 \mathrm{~kg}$ et $10 \mathrm{~kg}$ en moyenne par jour de pêche). Il est à signaler que le monofilament donne de meilleurs résultats que les autres filets dormants durant la mauvaise période.

La pêche au lac Albert est artisanale. Pour avoir accès au lac il faut avoir un permis de pêche mais malheureusement 55,8 à $69,1 \%$ de pêcheurs seulement détiennent le permis de pêche livrés par les services compétents et les autres sont considérés comme armateurs clandestins selon les résultats de nos enquêtes. Le montant des investissements pour une unité de pêche est fonction du type d'engin (notamment type de filet, type de moteur pour la navigation). La senne reste le filet le plus coûteux (2594,12 \$ $\pm 1361,71 \$)$ (Tableau 9). Seulement $28,9 \%$ des pêcheurs utilisent le moteur lors de la pêche. Les unités de pêche utilisant la senne, les filets dormants de surface ou de fond sont celles qui utilisent les moteurs pendant la pêche

Quant aux taxes, il ressort clairement, Tableau 10, que certains pêcheurs ne payent pas les taxes prévues. Ceci s'explique par le fait que, d'une part, certains camps de pêche comme Koga ne sont pas reconnus par le service d'Agriculture et les pêcheurs de ces camps ne peuvent pas avoir accès, officiellement aux permis et contrôle de pêche ; d'autre part, la faiblesse des services étatiques et la difficulté d'accès aux camps sont à la base de manque de suivi et de contrôle régulier des taxes. Il sied de signaler ici que les engins de pêche non autorités, bien qu'identifiés et utilisés ne sont jamais enregistrés par le service de camp et par conséquence ne paient aucune taxe.

Hormis les taxes et les frais investis dans l'achat des équipements de pêche, en fonction des engins utilisés, les activités de pêche impliquent des coûts à supporter à chaque sortie de pêche. Ceux qui utilisent les moteurs et qui représentent autour de 30\% dépensent plus que les autres catégories. La somme des dépenses pour la senne tournante (carburant, ration et autres dépenses) tourne autour de 37,21 \$ en moyenne. La pêche aux filets dormants de surface et de fond fait appel à un montant ne dépassant pas 23,35 \$ en moyenne quand on a recours à un moteur (Tableau 11).

Après la pêche, 61,4 à 74,2\% sont vendus frais directement après la pêche au niveau des plages, 37,1 à 50,7\% sont salés et le reste est soit fumé $(16 \%$ à $7,2 \%)$ ou soit conservé au froid ( 0 à $3,1 \%)$. Il sied de signaler que les pêcheurs se trouvant dans les camps Rukwanzi I, II, Matete, Kanana, Koga, procèdent directement au salage de leurs poissons car ils n'ont pas la possibilité 
d'écouler le frais suite à l'inaccessibilité et à la distance de leurs camps par rapport au marché.

On remarque que le prix du poisson par $\mathrm{kg}$ varie d'un camp de pêche à l'autre d'une part et d'autre part dépendant de l'abondance des captures.

L'analyse du Tableau 12 montre que lors de la bonne période de capture, $58,5 \%$ des poissons pêchés sont vendus à un prix variant entre 1,57 et $1,88 \$ / \mathrm{kg}$ et $33,2 \%$ à un prix autour de 1,25 à $1,56 \$ / \mathrm{kg}$. C'est dans les camps de pêche de Kanana, Tagba, Rukwanzi 1 et 2 et à Koga que l'on vend généralement à un prix plus faible.

Lorsque le poisson devient rare (baisse de la capture), le prix augmente (Tableau 13). $19,5 \%, 61,5 \%$ et $19,5 \%$ des poissons pêchés sont vendus respectivement de 1,89 à 2,19 $\$ / \mathrm{kg}, 2,2$ à $2,5 \$ / \mathrm{kg}$ et $2,51 \$ / \mathrm{kg}$. Il se remarque que le poisson coûte bon marché généralement dans les camps Kanana, Rukwanzi I et II, Tagba et Ndrigi toute la période de l'année (pendant la période faible et de grande capture) alors qu'il reste élevé dans les camps de pêche Mugumba, Muchanga et camp port qui sont, du reste, des camps plus accessibles et dont l'écoulement des produits de pêche est plus facile.

Malgré la grande variation des captures et revenus par unité de pêche (équipe de pêche), il ressort clairement de la Figure 4 qu'il existe une corrélation positive entre les deux. Les coefficients de corrélation linéaire sont de $+0,91$ et $+0,81$ respectivement en bonne et en mauvaise période de capture.

La comparaison du revenu individuel moyen des pêcheurs ouvriers en fonction d'engins de pêche utilisés montre une différence $(\mathrm{F}=1,32 ; 1-\mathrm{p}=76,73 \%$ en bonne période et $\mathrm{F}=2,33 ; 1-\mathrm{p}=98,14 \%$ ) en mauvaise période de pêche). L'analyse du Tableau 14 montre que la pêche à la nasse donne un bon rendement individuel après dépense pendant la bonne et la mauvaise période de pêche $(31,68$ $\pm 15 \$$ et $4,4 \pm 2,6 \$$ ) suivi de la senne tournante $(27,75 \pm 22,4 \$$ et $2,54 \pm 3,2 \$)$. L'épervier est le moins productif en terme de rendement que les autres filets de pêche $(10,7 \pm 5,3 \$$ et $3,28 \pm$ 1,9 \$). Lors des prises faibles, la senne tournante donne un revenu individuel plus faible que les autres engins de pêche avec parfois le risque de travailler à perte comme le montre le même tableau. Etant donné que le revenu total est divisé équitablement, après soustraction des dépenses liées à la pêche, entre les groupes d'ouvriers pêcheurs et l'armateur, il sied de signaler que la senne donne des bons rendements pour l'armateur que les autres engins de pêche car il faut multiplier le montant individuel par l'équipage pour déduire le revenu d'armateur $(27,75 \$$ x 10,47 $=290,54 \$$ en moyenne pendant la bonne période et $2,34 \mathrm{x}$ $10,47=24,5 \$$ pendant la mauvaise période de pêche).

\section{Causes de la baisse des captures et voies de sortie}

Les résultats de nos enquêtes montrent $83,4 \%$ pensent que la pêche est toujours rentable $($ Chi2 $=186,87 ; \mathrm{ddl}=1 ; 1-\mathrm{p}=>$ 99,99\%) malgré la dimension de la capture moyenne. Les causes de la diminution de la capture sont attribuées selon la plupart de pêcheurs, $($ Chi2 $=56,18 ;$ ddl $=10 ; 1-\mathrm{p}=>$ $99,99 \%$ ), à l'émergence des mauvais engins et techniques de pêche $(78,4 \%)$, à l'augmentation de l'effort de pêche $(54,8 \%)$ et à la non application de la réglementation de la pêche se traduisant par l'occupation des zones de frayères, la destruction des habitats, la pêche des poissons immatures de la pêche, la pêche illicite $(47,2 \%)$.

Pour pallier aux multiples problèmes identifiés comme causes de diminution de la capture, la majorité de pêcheurs (Chi2 = 901,$81 ; \mathrm{ddl}=10 ; 1-\mathrm{p}=>99,99 \%$ ) pensent qu'il faut d'abord éradiquer les mauvais engins et technique de pêche $(73,9 \%)$, l'implication effective et le sérieux des services étatiques chargées de réglementer la pêche $(63,8 \%)$, réduire l'effort de pêche $(56,5 \%)$ et mise à jour et application de la réglementation de la pêche $(48,2 \%)$. 
Tableau 1: Répartition des pêcheurs de la partie sud-ouest du lac Albert en fonction de leur tribu.

\begin{tabular}{rlrrr}
\hline & & \multicolumn{3}{c}{ Intervalle de confiance à 95\% } \\
$\mathbf{N}^{\circ}$ & Tribu & Pourcentage & Inférieur & Supérieur \\
\hline 1 & Alur & 34,7 & 29,9 & 39 \\
2 & Ngiti & 14,2 & 11,1 & 17,2 \\
3 & Lendu & 6,6 & 4,6 & 9,1 \\
4 & Hema & 13,9 & 10,9 & 17,2 \\
5 & Mungelema & 7,6 & 5,3 & 10,4 \\
6 & Bira & 0,5 & 0 & 1,3 \\
7 & Ngengere & 4,6 & 2,8 & 6,6 \\
8 & Muta linga & 0,8 & 0 & 1,8 \\
9 & Mbuza & 0,3 & 0 & 0,8 \\
10 & Lokele & 1 & 0,3 & 2,3 \\
11 & Nande & 6,3 & 4,1 & 8,6 \\
12 & Muhunde & 1,5 & 0,5 & 2,8 \\
13 & Logo & 2,3 & 1 & 3,8 \\
14 & Shi_Havu & 1,5 & 0,5 & 2,8 \\
15 & Budu & 0,8 & 0 & 1,8 \\
16 & Mubongo & 1,5 & 0,5 & 2,8 \\
17 & Rega & 0,3 & 0 & 0,8 \\
18 & Kaliko & 0,8 & 0 & 1,8 \\
19 & Mubudu & 1 & 0,3 & 2 \\
\hline
\end{tabular}

Tableau 2 : Age moyen des pêcheurs selon le camp de pêche au S-O du lac Albert.

\begin{tabular}{|c|c|c|c|}
\hline \multirow[b]{2}{*}{ Nom camp de pêche } & \multirow[b]{2}{*}{ Âge moyen pêcheur (ans) } & \multicolumn{2}{|c|}{ Intervalle de confiance à $95 \%$} \\
\hline & & Inférieur (ans) & Supérieur (ans) \\
\hline Camp port & $33,5 \pm 8,23$ & 30,32 & 36,64 \\
\hline Drigi & $34,5 \pm 15,08$ & 27,53 & 42,53 \\
\hline Kanana & $39,5 \pm 12,74$ & 32,94 & 45,73 \\
\hline Katho & $46,3 \pm 12,99$ & 40,3 & 52,12 \\
\hline Kiza & $37,1 \pm 6,77$ & 32,75 & 42,13 \\
\hline Koga & $35,8 \pm 9,2$ & 32,64 & 38,83 \\
\hline Lako & $37,1 \pm 11,1$ & 33,74 & 41 \\
\hline Malonga & $33,1 \pm 11,88$ & 29,23 & 36,86 \\
\hline Matete & $34,1 \pm 12,22$ & 30 & 38,35 \\
\hline Muchanga & $36,8 \pm 12,57$ & 32,68 & 41,15 \\
\hline Mugumba & $29,1 \pm 9,97$ & 25,4 & 32,56 \\
\hline Nyamavi & $33,2 \pm 14,46$ & 26,36 & 40,5 \\
\hline RukwanziI & $29,9 \pm 8,85$ & 27,38 & 32,69 \\
\hline RukwanziII & $32,9 \pm 9,35$ & 28,27 & 37,93 \\
\hline Tagba & $32,5 \pm 11,63$ & 28,58 & 36,32 \\
\hline Talimwana & $38,5 \pm 12,99$ & 31,92 & 45,85 \\
\hline Moyenne générale & $34,5 \pm 11,62$ & 33,45 & 35,63 \\
\hline
\end{tabular}




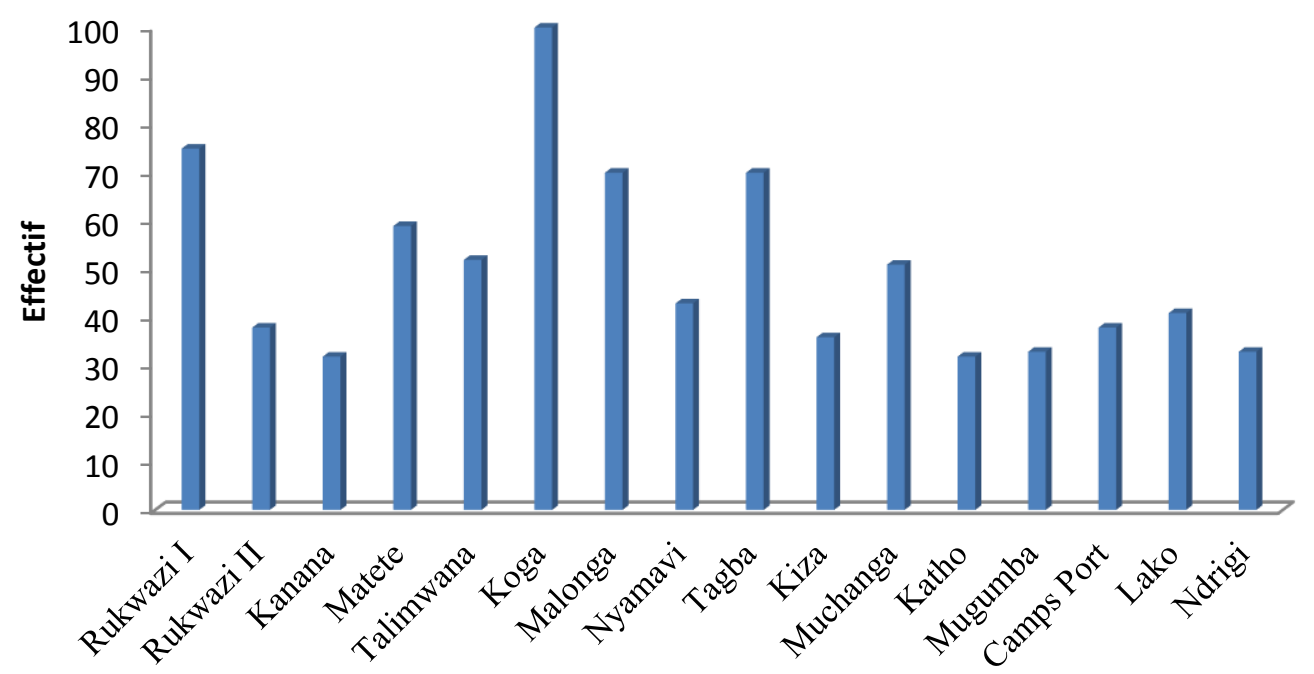

Figure 2 : Nombre des armateurs par camp de pêche au S-O du lac Albert.

Tableau 3 : Statistique d'engins de pêche en fonction des camps de pêche.

\begin{tabular}{|c|c|c|c|c|c|c|c|c|}
\hline $\begin{array}{l}\text { Camps de } \\
\text { pêche }\end{array}$ & 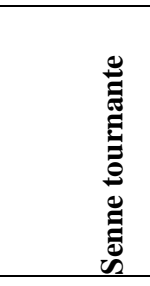 & 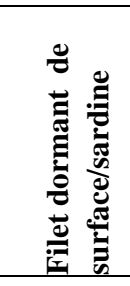 & 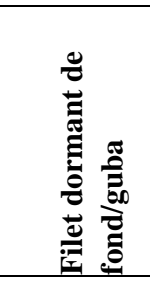 & 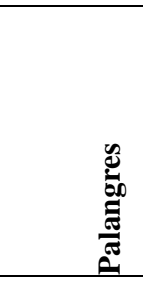 & 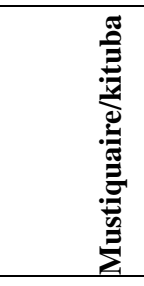 & $\begin{array}{l}\tilde{y} \\
. \\
\tilde{c} \\
\tilde{z} \\
\end{array}$ & 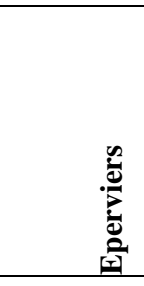 & 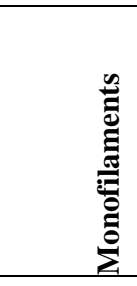 \\
\hline Camps Port & 8 & 9 & 25 & 4 & 0 & 0 & 11 & 0 \\
\hline Kanana & 0 & 19 & 22 & 8 & 1 & 0 & 0 & 5 \\
\hline Katho & 1 & 9 & 11 & 2 & 0 & 0 & 8 & 8 \\
\hline Kiza & 3 & 12 & 28 & 5 & 0 & 0 & 20 & 16 \\
\hline Koga & 17 & 66 & 50 & 15 & 3 & 50 & 0 & 30 \\
\hline Lako & 0 & 18 & 29 & 1 & 0 & 0 & 0 & 8 \\
\hline Malonga & 19 & 42 & 16 & 4 & 0 & 0 & 0 & 8 \\
\hline Matete & 37 & 14 & 9 & 5 & 1 & 0 & 0 & 10 \\
\hline Muchanga & 7 & 35 & 18 & 5 & 0 & 0 & 0 & 7 \\
\hline Mugumba & 15 & 8 & 8 & 6 & 1 & 0 & 0 & 0 \\
\hline Ndrigi & 0 & 20 & 9 & 8 & 0 & 0 & 2 & 2 \\
\hline Nyamavi & 6 & 18 & 32 & 13 & 5 & 5 & 0 & 5 \\
\hline Rukwazi I & 16 & 51 & 16 & 36 & 2 & 0 & 0 & 2 \\
\hline Rukwazi II & 3 & 21 & 12 & 10 & 2 & 0 & 0 & 3 \\
\hline Tagba & 17 & 20 & 15 & 8 & 0 & 20 & 12 & 75 \\
\hline Talimwana & 0 & 58 & 26 & 28 & 4 & 10 & 8 & 16 \\
\hline Moyenne & 9,313 & 26,250 & 20,375 & 9,875 & 1,188 & 5,313 & 3,813 & 12,188 \\
\hline Ecart-type & 10,229 & 18,441 & 11,087 & 9,500 & 1,601 & 13,098 & 6,134 & 18,382 \\
\hline Total $\%$ & 10,5449 & 29,724 & 23,0715 & 11,1819 & 1,34466 & 6,01557 & 4,31706 & 13,8004 \\
\hline
\end{tabular}




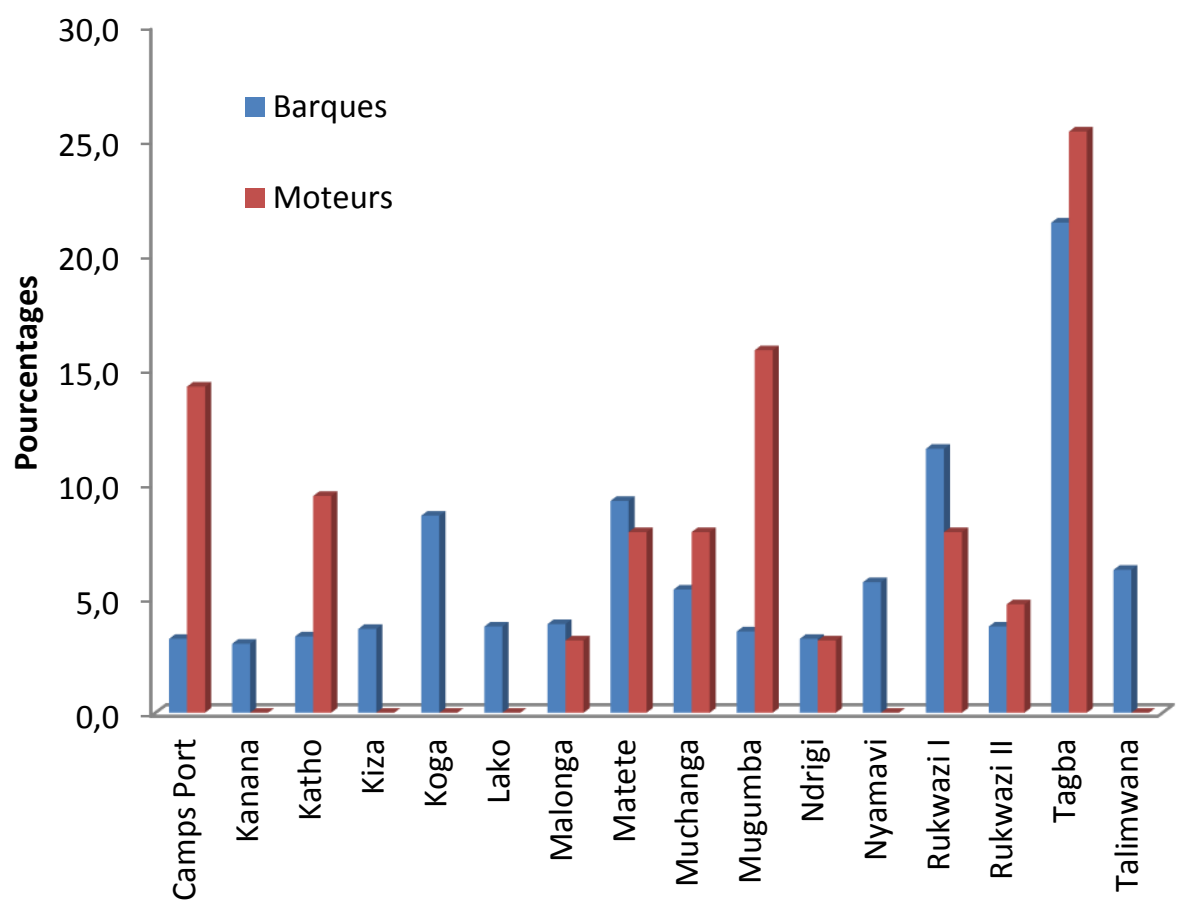

Figure 3 : Répartition des barques et moteurs dans différents camps de pêche.

Tableau 4 : Caractéristiques d'une unité de pêche en fonction du type d'engin au S-O du lac Albert.

\begin{tabular}{|c|c|c|c|c|c|c|c|}
\hline & $\begin{array}{l}\text { Senne } \\
\text { tournante }\end{array}$ & $\begin{array}{l}\text { Filet dormant de } \\
\text { surface }\end{array}$ & $\begin{array}{l}\text { Filet dormant } \\
\text { de fond }\end{array}$ & $\begin{array}{l}\text { Filet dormant } \\
\text { monofilament }\end{array}$ & Palangre & Nasses & Epervier \\
\hline Nombre membres d'équipage & $10,47 \pm 2,65$ & $2,49 \pm 0,79$ & $2,03 \pm 0,35$ & $2,09 \pm 0,52$ & $2,1 \pm 0,3$ & $1,59 \pm 0,5$ & $1,88 \pm 0,5$ \\
\hline Nombre de barques & $1,6 \pm 0,49$ & $1,23 \pm 0,68$ & $1,23 \pm 0,8$ & $1,08 \pm 0,27$ & $1,05 \pm 0,2$ & $1,05 \pm 02$ & $1 \pm 00$ \\
\hline Longueur moyenne de barque(m) & $7,41 \pm 4,4$ & $4,82 \pm 1,47$ & $4,41 \pm 0,78$ & $3,92 \pm 0,83$ & $4,17 \pm 0,54$ & $3,78 \pm 0,54$ & $4,04 \pm 0,49$ \\
\hline Longueur filet(m) & $337,41 \pm 120,1$ & $766,26 \pm 536$ & $1053,05 \pm 520$ & $316,8 \pm 120,3$ & $1343,67 \pm 832,5$ & - & $4,75 \pm 1,7$ \\
\hline Nombre de sorties/semaine & $5,51 \pm 1,59$ & $5,39 \pm 1,33$ & $5,46 \pm 1,39$ & $5,12 \pm 1,68$ & $4,95 \pm 2,34$ & $6,81 \pm 0,87$ & $5,63 \pm 1,41$ \\
\hline $\begin{array}{l}\text { Nombre de fois de jet de filet par } \\
\text { sortie) }\end{array}$ & $7,73 \pm 3,47$ & $1,41 \pm 0,38$ & $1,2 \pm 0,21$ & $9,88 \pm 12,32$ & $1 \pm 00$ & $1 \pm 00$ & $40 \pm 39,71$ \\
\hline Temps d'une capture (minutes) & $87,19 \pm 31,64$ & $415,63 \pm 239,14$ & $578,75 \pm 237,9$ & $60 \pm 31,6$ & $501,1 \pm 217,9$ & $918,86 \pm 339,7$ & $11 \pm 6,9$ \\
\hline
\end{tabular}


Tableau 5 : Raisons de l'augmentation des captures pendant une période de l'année au S-O du lac Albert.

\begin{tabular}{lccccccc}
\hline $\begin{array}{l}\text { Type de filet/Raisons } \\
\text { de hautes } \\
\text { captures/mois }\end{array}$ & $\begin{array}{l}\text { Senne } \\
\text { tournante }\end{array}$ & $\begin{array}{l}\text { Filet } \\
\text { dormant de } \\
\text { surface }\end{array}$ & $\begin{array}{l}\text { Filet } \\
\text { dormant de } \\
\text { fond }\end{array}$ & $\begin{array}{l}\text { Filet dormant } \\
\text { monofilament }\end{array}$ & Palangre & Nasses & Epervier \\
\hline $\begin{array}{l}\text { Saison de pluie } \\
\text { Fort vent provoquant }\end{array}$ & $64,50 \%$ & $54,30 \%$ & $63,20 \%$ & $62,20 \%$ & $76,20 \%$ & $59,10 \%$ & $75,00 \%$ \\
des vagues & $42,10 \%$ & $62,90 \%$ & $46,20 \%$ & $54,10 \%$ & $42,90 \%$ & $27,30 \%$ & $25,00 \%$ \\
Période après ouverture & $17,80 \%$ & $15,70 \%$ & $13,70 \%$ & $18,90 \%$ & $4,80 \%$ & $9,10 \%$ & $37,50 \%$ \\
$\begin{array}{l}\text { Zones riches ou } \\
\text { frayères }\end{array}$ & $9,30 \%$ & $5,70 \%$ & $4,30 \%$ & $4,10 \%$ & $0,00 \%$ & $4,50 \%$ & $50,00 \%$ \\
$\begin{array}{l}\text { Période capture et sans } \\
\text { trop de vagues }\end{array}$ & $15,90 \%$ & $17,10 \%$ & $11,10 \%$ & $20,30 \%$ & $0,00 \%$ & $0,00 \%$ & $0,00 \%$ \\
$\begin{array}{l}\text { Période après les } \\
\text { vagues }\end{array}$ & $15,00 \%$ & $21,40 \%$ & $15,40 \%$ & $17,60 \%$ & $14,30 \%$ & $9,10 \%$ & $37,50 \%$ \\
$\begin{array}{l}\text { Période après la saison } \\
\text { des pluies }\end{array}$ & $12,10 \%$ & $17,10 \%$ & $8,50 \%$ & $6,80 \%$ & $9,50 \%$ & $13,60 \%$ & $0,00 \%$ \\
$\begin{array}{l}\text { Diminution de niveau } \\
\text { d'eau }\end{array}$ & $25,20 \%$ & $18,60 \%$ & $22,20 \%$ & $36,50 \%$ & $14,30 \%$ & $63,60 \%$ & $62,50 \%$ \\
$\begin{array}{l}\text { Augmentation de } \\
\text { niveau d'eau }\end{array}$ & $27,10 \%$ & $21,40 \%$ & $32,50 \%$ & $17,60 \%$ & $19,00 \%$ & $40,90 \%$ & $37,50 \%$ \\
Absence de la lumière & $1,90 \%$ & $1,40 \%$ & $0,00 \%$ & $0,00 \%$ & $0,00 \%$ & $0,00 \%$ & $0,00 \%$ \\
\hline
\end{tabular}

Tableau 6 : Choix des engins et techniquees de pêche en fonction des sites de pêche au S-O du lac Albert.

\begin{tabular}{|c|c|c|c|c|c|c|c|c|}
\hline $\begin{array}{l}\text { Sites de prédilection } \\
\text { de pêche/Type de } \\
\text { filet }\end{array}$ & $\begin{array}{l}\text { Senne } \\
\text { tournante }\end{array}$ & $\begin{array}{l}\text { Filet } \\
\text { dormant de } \\
\text { surface }\end{array}$ & $\begin{array}{l}\text { Filet } \\
\text { dormant de } \\
\text { fond }\end{array}$ & $\begin{array}{l}\text { Filet dormant } \\
\text { monofilament }\end{array}$ & Palangre & Nasses & Epervier & TOTAL \\
\hline Zone littorale & $13,00 \%$ & $15,00 \%$ & $30,00 \%$ & $20,00 \%$ & $7,00 \%$ & $15,00 \%$ & $6,00 \%$ & $100 \%$ \\
\hline Zone de frayère & $21,20 \%$ & $3,00 \%$ & $21,20 \%$ & $27,30 \%$ & $12,10 \%$ & $6,10 \%$ & $12,10 \%$ & $100 \%$ \\
\hline $\begin{array}{l}\text { Zone riche en } \\
\text { macrophytes }\end{array}$ & $27,00 \%$ & $4,80 \%$ & $27,00 \%$ & $27,00 \%$ & $4,80 \%$ & $12,70 \%$ & $3,20 \%$ & $100 \%$ \\
\hline $\begin{array}{l}\text { Zone de grandes } \\
\text { profondeurs }\end{array}$ & $42,20 \%$ & $18,00 \%$ & $24,60 \%$ & $11,40 \%$ & $3,80 \%$ & $0,00 \%$ & $1,40 \%$ & $100 \%$ \\
\hline $\begin{array}{l}\text { Zone de faibles } \\
\text { profondeurs }\end{array}$ & $16,10 \%$ & $19,90 \%$ & $34,90 \%$ & $24,70 \%$ & $7,00 \%$ & $6,50 \%$ & $1,10 \%$ & $100 \%$ \\
\hline Tous les sites & $38,90 \%$ & $11,10 \%$ & $27,80 \%$ & $16,70 \%$ & $5,60 \%$ & $8,30 \%$ & $0,00 \%$ & $100 \%$ \\
\hline Pas de critère & $0,00 \%$ & $40,00 \%$ & $60,00 \%$ & $0,00 \%$ & $0,00 \%$ & $0,00 \%$ & $0,00 \%$ & $100 \%$ \\
\hline
\end{tabular}


Tableau 7 : Capture des espèces en fonction des engins et techniques de pêche au S-O du lac Albert.

\begin{tabular}{|c|c|c|c|c|c|c|c|c|}
\hline $\begin{array}{l}\text { Type de filet/Espèces à haute } \\
\text { valeur commerciale }\end{array}$ & $\begin{array}{l}\text { Senne } \\
\text { tournante }\end{array}$ & $\begin{array}{l}\text { Filet dormant de } \\
\text { surface }\end{array}$ & $\begin{array}{l}\text { Filet dormant } \\
\text { de fond }\end{array}$ & $\begin{array}{l}\text { Filet dormant } \\
\text { monofilament }\end{array}$ & Palangre & Nasse & Epervier & TOTAL \\
\hline Hydrocynus forskhalii & $3,7 \%$ & $15,7 \%$ & $20,5 \%$ & $4,1 \%$ & $0,0 \%$ & $0,0 \%$ & $0,0 \%$ & $10,3 \%$ \\
\hline Hydrocynus vittatus & $6,5 \%$ & $40,0 \%$ & $20,5 \%$ & $8,1 \%$ & $0,0 \%$ & $0,0 \%$ & $0,0 \%$ & $15,6 \%$ \\
\hline Alestes baremoze & $1,9 \%$ & $57,1 \%$ & $4,3 \%$ & $0,0 \%$ & $0,0 \%$ & $0,0 \%$ & $0,0 \%$ & $11,1 \%$ \\
\hline Alestes dentex dentex & $1,9 \%$ & $55,7 \%$ & $5,1 \%$ & $0,0 \%$ & $0,0 \%$ & $0,0 \%$ & $0,0 \%$ & $11,1 \%$ \\
\hline Labeo coubie & $3,7 \%$ & $0,0 \%$ & $3,4 \%$ & $1,4 \%$ & $0,0 \%$ & $0,0 \%$ & $0,0 \%$ & $2,3 \%$ \\
\hline Barbus bynni bynni & $0,9 \%$ & $0,0 \%$ & $0,0 \%$ & $0,0 \%$ & $0,0 \%$ & $0,0 \%$ & $0,0 \%$ & $0,3 \%$ \\
\hline Bagrus spp. & $33,6 \%$ & $5,7 \%$ & $19,7 \%$ & $21,6 \%$ & $42,9 \%$ & $0,0 \%$ & $37,5 \%$ & $21,7 \%$ \\
\hline Auchenoglanis occidentalis & $12,1 \%$ & $10,0 \%$ & $9,4 \%$ & $18,9 \%$ & $9,5 \%$ & $0,0 \%$ & $12,5 \%$ & $10,3 \%$ \\
\hline Clarias gariepinus & $8,4 \%$ & $1,4 \%$ & $9,4 \%$ & $4,1 \%$ & $57,1 \%$ & $4,5 \%$ & $0,0 \%$ & $9,3 \%$ \\
\hline Synodontis schall & $20,6 \%$ & $5,7 \%$ & $18,8 \%$ & $14,9 \%$ & $4,8 \%$ & $0,0 \%$ & $12,5 \%$ & $14,3 \%$ \\
\hline Synodontis frontosus & $22,4 \%$ & $2,9 \%$ & $18,8 \%$ & $12,2 \%$ & $4,8 \%$ & $0,0 \%$ & $12,5 \%$ & $14,3 \%$ \\
\hline Synodontis nigrita & $2,8 \%$ & $0,0 \%$ & $0,0 \%$ & $0,0 \%$ & $0,0 \%$ & $0,0 \%$ & $0,0 \%$ & $0,8 \%$ \\
\hline Lates macrophthalmus & $79,4 \%$ & $24,3 \%$ & $52,1 \%$ & $40,5 \%$ & $14,3 \%$ & $9,1 \%$ & $75,0 \%$ & $48,7 \%$ \\
\hline Lates niloticus & $58,9 \%$ & $2,9 \%$ & $15,4 \%$ & $8,1 \%$ & $0,0 \%$ & $4,5 \%$ & $0,0 \%$ & $22,6 \%$ \\
\hline Coptodon zillii & $45,8 \%$ & $15,7 \%$ & $24,8 \%$ & $66,2 \%$ & $4,8 \%$ & $68,2 \%$ & $37,5 \%$ & $37,2 \%$ \\
\hline Sarotherodon galilaeus & $41,1 \%$ & $7,1 \%$ & $23,9 \%$ & $51,4 \%$ & $14,3 \%$ & $81,8 \%$ & $12,5 \%$ & $33,2 \%$ \\
\hline Oreochromis spp. & $55,1 \%$ & $4,3 \%$ & $28,2 \%$ & $63,5 \%$ & $19,0 \%$ & $95,5 \%$ & $62,5 \%$ & $41,5 \%$ \\
\hline Brycinus sp & $21,5 \%$ & $4,3 \%$ & $20,5 \%$ & $21,6 \%$ & $14,3 \%$ & $54,5 \%$ & $0,0 \%$ & $18,8 \%$ \\
\hline
\end{tabular}

Tableau 8 : Variation de la Capture par Unité d'Effort de Pêche (CUEP) en fonction des engins de pêche au S-O du lac Albert.

\begin{tabular}{lcc}
\hline Engins de pêche & Qté bonne période (kg/jour) & Qté mauvaise période (kg/jour) \\
\hline Senne tournante & $183,18 \pm 49,02$ & $20,89 \pm 12,94$ \\
Filet dormant de surface & $62,79 \pm 23,24$ & $10,21 \pm 5,49$ \\
Filet dormant de fond & $62,74 \pm 21,07$ & $10,06 \pm 6,63$ \\
Filet dormant monofilament & $48,99 \pm 22,68$ & $12,3 \pm 7,99$ \\
Palangre & $37,62 \pm 19,21$ & $10,48 \pm 20,91$ \\
Nasses & $20 \pm 6,9$ & $6,32 \pm 3,2$ \\
Epervier & $10,75 \pm 13,56$ & $4,88 \pm 2,47$ \\
\hline
\end{tabular}


Tableau 9 : Prix des engins de pêche au S-O du lac Albert.

\begin{tabular}{llccc}
\hline $\mathbf{N}^{\circ}$ & Libellé & Type & Qté & Moyenne Prix $\mathbf{( \$ )}$ \\
\hline 1 & Prix Barque & Bois & 1 & $320,71 \pm 146,08$ \\
2 & Moteur & Yamaha $(15$ ou $9 \mathrm{CV})$ & 1 & $2301,96 \pm 930,86$ \\
3 & Filet senne tournante & Soies & 1 & $2594,12 \pm 1361,71$ \\
4 & Filet maillant de fond & Soies & 1 & $293,79 \pm 200,24$ \\
5 & Filet maillant de surface & Soies & 1 & $274,55 \pm 110,55$ \\
6 & Palangre & Nylon & 1 & $42,66 \pm 59,21$ \\
7 & Monofilament & Nylon & 1 & $26,93 \pm 10,83$ \\
8 & Nasse & Files métalliques & 1 & $15,25 \pm 14,88$ \\
\hline
\end{tabular}

Tableau 10 : Structure des différentes taxes au S-O du lac Albert.

\begin{tabular}{clcccll}
\hline $\mathbf{N}^{\circ}$ & Libelle & Moyenne Prix $(\$)$ & $\mathbf{M i n}(\mathbf{\$})$ & $\mathbf{M a x}(\mathbf{\$})$ & Service bénéficiaire & Période \\
\hline 1 & Numéro barque & $29,12 \pm 20,01$ & 0 & 50 & Service Maritime & une fois \\
2 & Taxe barque & $8,66 \pm 10,88$ & 0 & 37,5 & Service Maritime & Annuelle \\
3 & Permis de pêche & $39,62 \pm 23,76$ & 0 & 50 & Division d'agriculture & Annuelle \\
4 & Taxe collectivité & $21,6 \pm 11,58$ & 0 & 55 & Collectivité & Trimestrielle \\
5 & Mesurage filet & $4,78 \pm 2,5$ & 0 & 9,36 & Camp de pêche & Trimestrielle \\
6 & $\begin{array}{l}\text { Ration militaire } \\
\text { (Marin) }\end{array}$ & $1,24 \pm 0,9$ & 0 & 3,13 & Service Maritime & Journalière \\
7 & Contrôle agri-pêche & $4,2 \pm 3,57$ & 0 & 10 & Division d'agriculture & Trimestrielle \\
8 & Taxe de navigation & $4,05 \pm 9,33$ & 0 & 50 & Service Maritime & Annuelle \\
9 & Taxe moteur & $5,4 \pm 14,85$ & 0 & 50 & Service Maritime & Annuelle \\
\hline
\end{tabular}

Tableau 11 : Coût moyen lors d'une sortie de pêche par Unité d'effort de pêche au S-O du lac Albert.

\begin{tabular}{llllcccc}
\hline Catégorie & $\begin{array}{l}\text { Senne } \\
\text { tournante }\end{array}$ & $\begin{array}{l}\text { Filet } \\
\text { dormant } \\
\text { de surface }\end{array}$ & $\begin{array}{l}\text { Filet } \\
\text { dormant } \\
\text { de fond }\end{array}$ & $\begin{array}{l}\text { Filet dormant } \\
\text { monofilament }\end{array}$ & Palangre & Nasses & Epervier \\
\hline $\begin{array}{l}\text { Quantité } \\
\text { carburant/litre }\end{array}$ & $23,55 \pm 4,77$ & $18,21 \pm 6,08$ & $18,29 \pm 7$ & 0 & 0 & 0 & 0 \\
$\begin{array}{l}\text { Coût ration } \\
\begin{array}{l}\text { Coût dépense hors } \\
\text { ration }\end{array}\end{array}$ & $9,87 \pm 5,56$ & $2,8 \pm 1,89$ & $3,79 \pm 1,23$ & $2,71 \pm 0,72$ & $2,17 \pm 1,7$ & $0,61 \pm 0,99$ & $2,42 \pm 1,3$ \\
\hline
\end{tabular}


Tableau 12 : Variation de prix des poissons lors de la bonne saison en fonction du camp de pêche au S-O du lac Albert.

\begin{tabular}{|c|c|c|c|c|c|c|}
\hline Camp de pêche & $\begin{array}{l}\text { De } 1,25 \text { à } \\
1,56 \$ / k g\end{array}$ & $\begin{array}{l}\text { De } 1,57 \text { à } \\
1,88 \$ / k g\end{array}$ & $\begin{array}{l}\text { De } 1,89 \text { à } \\
2,19 \$ / k g\end{array}$ & $\begin{array}{c}\text { De } 2,2 \text { à } 2,5 \\
\$ / \mathrm{kg} \\
\end{array}$ & $\begin{array}{c}2,51 \$ / \mathrm{kg} \\
\text { et plus }\end{array}$ & TOTAL \\
\hline Camp port & $0,0 \%$ & $88,9 \%$ & $11,1 \%$ & $0,0 \%$ & $0,0 \%$ & $100 \%$ \\
\hline Kanana & $100,0 \%$ & $0,0 \%$ & $0,0 \%$ & $0,0 \%$ & $0,0 \%$ & $100 \%$ \\
\hline Katho & $30,0 \%$ & $70,0 \%$ & $0,0 \%$ & $0,0 \%$ & $0,0 \%$ & $100 \%$ \\
\hline Kiza & $20,0 \%$ & $70,0 \%$ & $10,0 \%$ & $0,0 \%$ & $0,0 \%$ & $100 \%$ \\
\hline Koga & $58,3 \%$ & $41,7 \%$ & $0,0 \%$ & $0,0 \%$ & $0,0 \%$ & $100 \%$ \\
\hline Lako & $11,1 \%$ & $88,9 \%$ & $0,0 \%$ & $0,0 \%$ & $0,0 \%$ & $100 \%$ \\
\hline Malonga & $14,3 \%$ & $64,3 \%$ & $21,4 \%$ & $0,0 \%$ & $0,0 \%$ & $100 \%$ \\
\hline Matete & $22,7 \%$ & $77,3 \%$ & $0,0 \%$ & $0,0 \%$ & $0,0 \%$ & $100 \%$ \\
\hline Muchanga & $7,7 \%$ & $69,2 \%$ & $23,1 \%$ & $0,0 \%$ & $0,0 \%$ & $100 \%$ \\
\hline Mugumba & $9,1 \%$ & $63,6 \%$ & $27,3 \%$ & $0,0 \%$ & $0,0 \%$ & $100 \%$ \\
\hline Ndrigi & $37,5 \%$ & $62,5 \%$ & $0,0 \%$ & $0,0 \%$ & $0,0 \%$ & $100 \%$ \\
\hline Nyamavi & $0,0 \%$ & $91,7 \%$ & $8,3 \%$ & $0,0 \%$ & $0,0 \%$ & $100 \%$ \\
\hline RukwanziI & $52,6 \%$ & $47,4 \%$ & $0,0 \%$ & $0,0 \%$ & $0,0 \%$ & $100 \%$ \\
\hline RukwanziII & $90,0 \%$ & $10,0 \%$ & $0,0 \%$ & $0,0 \%$ & $0,0 \%$ & $100 \%$ \\
\hline Tagba & $50,0 \%$ & $28,6 \%$ & $21,4 \%$ & $0,0 \%$ & $0,0 \%$ & $100 \%$ \\
\hline Talimwana & $0,0 \%$ & $80,0 \%$ & $20,0 \%$ & $0,0 \%$ & $0,0 \%$ & $100 \%$ \\
\hline TOTAL & $33,20 \%$ & $58,50 \%$ & $8,30 \%$ & $0,00 \%$ & $0,00 \%$ & $100 \%$ \\
\hline
\end{tabular}

Tableau 13 : Variation de prix des poissons lors de la mauvaise saison en fonction du camp de pêche au S-O du lac Albert.

\begin{tabular}{|c|c|c|c|c|c|c|}
\hline $\begin{array}{l}\text { Camp de pêche/Prix } \\
\text { 1kg/faible capture/Fc }\end{array}$ & $\begin{array}{l}\text { De } 1,25 \text { à } \\
1,56 \$ / \mathrm{kg}\end{array}$ & $\begin{array}{l}\text { De } 1,57 \text { à } \\
1,88 \$ / k g\end{array}$ & $\begin{array}{l}\text { De 1,89 à } \\
2,19 \$ / \mathrm{kg} \\
\end{array}$ & $\begin{array}{c}\text { De } 2,2 \text { à } 2,5 \\
\$ / k g\end{array}$ & $\begin{array}{c}2,51 \$ / \mathrm{kg} \\
\text { et plus }\end{array}$ & TOTAL \\
\hline Camp port & $0,0 \%$ & $0,0 \%$ & $0,0 \%$ & $33,3 \%$ & $66,7 \%$ & $100 \%$ \\
\hline Kanana & $0,0 \%$ & $0,0 \%$ & $100,0 \%$ & $0,0 \%$ & $0,0 \%$ & $100 \%$ \\
\hline Katho & $0,0 \%$ & $0,0 \%$ & $20,0 \%$ & $80,0 \%$ & $0,0 \%$ & $100 \%$ \\
\hline Kiza & $0,0 \%$ & $0,0 \%$ & $0,0 \%$ & $90,0 \%$ & $10,0 \%$ & $100 \%$ \\
\hline Koga & $0,0 \%$ & $0,0 \%$ & $12,5 \%$ & $70,8 \%$ & $16,7 \%$ & $100 \%$ \\
\hline Lako & $0,0 \%$ & $0,0 \%$ & $0,0 \%$ & $88,9 \%$ & $11,1 \%$ & $100 \%$ \\
\hline Malonga & $0,0 \%$ & $0,0 \%$ & $14,3 \%$ & $64,3 \%$ & $21,4 \%$ & $100 \%$ \\
\hline Matete & $0,0 \%$ & $0,0 \%$ & $9,1 \%$ & $86,4 \%$ & $4,5 \%$ & $100 \%$ \\
\hline Muchanga & $0,0 \%$ & $0,0 \%$ & $15,4 \%$ & $38,5 \%$ & $46,2 \%$ & $100 \%$ \\
\hline Mugumba & $0,0 \%$ & $0,0 \%$ & $0,0 \%$ & $27,3 \%$ & $72,7 \%$ & $100 \%$ \\
\hline Ndrigi & $0,0 \%$ & $0,0 \%$ & $37,5 \%$ & $62,5 \%$ & $0,0 \%$ & $100 \%$ \\
\hline
\end{tabular}




\begin{tabular}{lllllll} 
Nyamavi & $0,0 \%$ & $0,0 \%$ & $8,3 \%$ & $66,7 \%$ & $25,0 \%$ & $100 \%$ \\
RukwanziI & $0,0 \%$ & $0,0 \%$ & $31,6 \%$ & $63,2 \%$ & $5,3 \%$ & $100 \%$ \\
RukwanziII & $0,0 \%$ & $0,0 \%$ & $40,0 \%$ & $60,0 \%$ & $0,0 \%$ & $100 \%$ \\
Tagba & $0,0 \%$ & $0,0 \%$ & $35,7 \%$ & $42,8 \%$ & $28,6 \%$ & $100 \%$ \\
Talimwana & $0,0 \%$ & $0,0 \%$ & $0,0 \%$ & $80,0 \%$ & $20,0 \%$ & $100 \%$ \\
\hline TOTAL & $0,0 \%$ & $0,0 \%$ & $19,5 \%$ & $61,5 \%$ & $19,5 \%$ & $100 \%$ \\
\hline
\end{tabular}
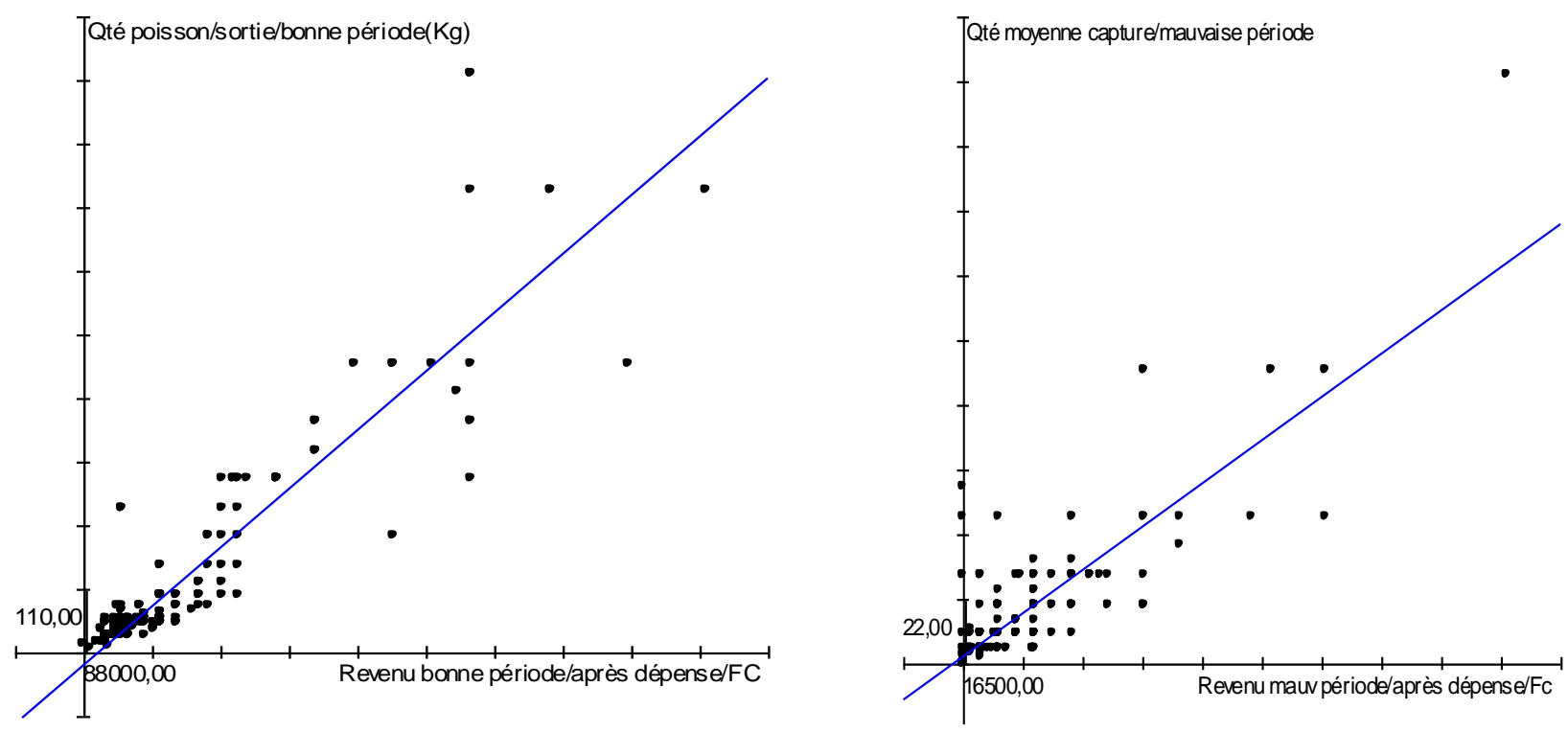

Figure 4: Corrélation linéaire de Pearson entre la quantité capturée (kg) et le revenu (Fr congolais) par unité d'effort de pêche ( $1 \$=1600 \mathrm{Fr}$ congolais).

Tableau 14 : Revenu individuel journalier par Unité d'effort de pêche au S-O du lac Albert.

\begin{tabular}{lcc}
\hline Engin de pêche & Bonne capture (\$) & Faible capture (\$) \\
\hline Senne tournante & $27,75 \pm 22,4$ & $2,54 \pm 3,2$ \\
Filet dormant de surface & $19,88 \pm 16,8$ & $2,71 \pm 3$ \\
Filet dormant de fond & $22,89 \pm 18,9$ & $4,1 \pm 2,3$ \\
Filet dormant monofilament & $21,6 \pm 19$ & $4,03 \pm 3,1$ \\
Palangre & $20,4 \pm 15,9$ & $3,57 \pm 2,8$ \\
Nasses & $31,68 \pm 15$ & $4,4 \pm 2,6$ \\
Epervier & $10,7 \pm 5,3$ & $3,28 \pm 1,9$ \\
\hline TOTAL & $20,6 \pm 16,2$ & $3,52 \pm 7,7$ \\
\hline
\end{tabular}




\section{DISCUSSION}

Le secteur de la pêche offre des retombées économiques considérables à la population de la province d'Ituri. La pêche est généralement pratiquée par les hommes du sexe masculin, dominés par la tribu Alur (29,9 à $39 \%$ dans la partie sud-ouest du lac en territoire d'Irumu). Le niveau d'instruction du cycle d'orientation ( 2 ans post-primaires) et du primaire domine parmi les pêcheurs (respectivement $42,6 \%$ et $39,3 \%$ ) qui sont mariés à plus de $70 \%$ et dont l'âge moyen est de 34,5 $\pm 11,1$ ans. Ces résultats sont proches de ceux trouvés par Mukabo et al. (2017) au lac Tanganyika; Tah et al. 2007 au lac Ayamé I. Ce qui signifie que la pêche au lac Albert est pratiquée par les personnes de faible niveau d'instruction et qui sont d'âge actif car cette activité nécessite beaucoup d'efforts physiques.

Quant aux engins de pêche, dans cette partie du lac, les techniques passives représentent plus de $80 \%$ des engins utilisés (les filets maillants de surface, de fond, la palangre, le monofilament et les nasses) contre moins de $20 \%$ des techniques actives données par la senne tournante. L'arrêté provincial de 2016 sur la réglementation de la pêche au lac Albert considère que la senne de plage, la senne tournante, les monofilaments, la pêche au tamtam et les nasses sont des engins prohibés. On constate une réduction considérable, en nombre de nasses (de 50\% à 5,99\%) et une progression positive des filets dormants de surface (de 7,64\% à 29,59\%) et de fond (de $12,73 \%$ à 22,97\%). Ce résultat laisserait croire que l'effort de la réglementation de la pêche qui se traduit par des patrouilles mixtes régulièrement faites par les services d'environnement et de la force navale est en train de donner des résultats positifs dans cette partie du lac. La pêche aux moustiquaires est faite dans le but de trouver généralement les appâts (Haplochromis spp.) pour la pêche à la ligne (palangre) alors qu'au lac Mai-Ndombe les moustiquaires sont utilisées pour la pêche illicite et ces dernières associées à d'autres filets à techniques actives comme la senne de plage détruisent la biodiversité de cet écosystème (Bongeba et Micha, 2013 ; Luhusu et Micha, 2013).

En comparant avec les engins de pêche utilisés aux lacs Edouard et Tanganyika (Mukabo et al., 2017; Balolebwami et al., 2018 et Mushagalusa et al., 2015), il ressort clairement que les techniques de pêche dans ces trois écosystèmes diffèrent. Au lac Tanganyika la pêche est basée sur les unités de pêche constituées soit de Catamaran (groupe de deux pirogues, un filet, un moteur ou des rames selon le cas employé par 4 à 8 personnes), soit d'Apollo constitué d'un groupe de 3 pirogues avec un filet et est généralement motorisé. Les 2 techniques de pêche se basent sur l'attraction des poissons par la lumière et la pêche se fait la nuit et les espèces visées sont Lates stapersii, Limnothrissa miodon et Stolothrissa tanganyicae. Au lac Edouard, la pêche emploie la ligne (palangre) et les filets dormants avec comme cibles Bagrus docmak (64\% de la capture) et Oreochromis niloticus (26\%). 7 pêcheurs constituent en moyenne une Unité de pêche. Contrairement, au lac Albert, les techniques et les engins de pêche sont très diversifiés (8 types d'engins inventoriés), la senne tournante semble être moins sélective et capture une diversité de poissons comme le montre le tableau 7 alors que les autres engins de pêche comme les filets dormants de surface et de fond ciblent respectivement la famille Alestidae avec ses 4 espèces et les espèces du genre Lates. Il est démontré par les autres chercheurs que la senne en généralement est moins sélective et a des conséquences négatives sur l'ichtyofaune et les habitats côtiers aquatiques (Hounsounou et al., 2013) et cela peut conduire à la surpêche et par conséquent au cycle vicieux de la pauvreté des pêcheurs (CRHOB, 2004 ; Hounsounou, 2011) mais cet engin reste le plus préféré des pêcheurs au lac Albert.

En termes de nombre de pirogues, l'unité de pêche utilise généralement 2 barques, parfois une seule avec une senne tournante d'une moyenne de 337,4 mètres de long (pouvant atteindre une longueur maximum de 1 kilomètre) ou un filet dormant de surface ou de fond pouvant atteindre 2 kilomètres de long (longueur moyenne respective de 766,26 \pm 536 
mètres et 1053,05 \pm 520 mètres). Chaque unité de pêche comprend autour de 8 à 10 pêcheurs pour la senne et 1 à 2 personnes pour les autres techniques de pêche (Tableau 4). Les activités de pêche se passent la journée et débute vers 4 ou 5 heures du matin et bien encore vers le coucher du soleil entre 16 et 17 heures comme au lac Edouard. La comparaison entre ces deux lacs montre, qu'en moyenne, la pêche dure 96 heures par semaine au lac Edouard contre 58 heures au lac Albert en mettant en concours la moyenne du nombre de fois qu'on jette le filet. Ainsi les activités de pêche couvrent une période de 116 jours par an, sensiblement inférieure à celle observée par Balole Bwami et al. (2018) au lac Edouard (soit 219,8 jours/an).

Parlant des captures par unité d'effort de pêche (CPUE), on constate une variabilité périodique des captures mais pendant la bonne période (mars, avril, août et novembre), elles sont bonnes $(183,18 \pm 49,02 \mathrm{~kg} / \mathrm{jour}$ pour la senne, $62,79 \pm 23,24$ et $62,74 \pm 21,07 \mathrm{~kg} / \mathrm{jour}$ respectivement pour les filets dormants de surface et de fond) que celles enregistrées aux lacs Tanganyika (Apollo 80 à $152 \mathrm{~kg} / \mathrm{jour}$ et Catamaran $50 \mathrm{~kg} / \mathrm{jour}$ par Mukabo et al. (2017), Taabo (une moyenne de la senne de plage de 59,52 kg/jour) et Edouard (moyenne de filet dormant et palangre est de 26,27 $\mathrm{kg}$ /jour) ; lors de la mauvaise période de pêche, les captures sont généralement inférieures à 20 $\mathrm{kg} / \mathrm{jour}$.

Le prix d'un $\mathrm{kg}$ varie beaucoup d'un milieu à l'autre, d'un pays à l'autre. Au Sénégal par exemple le prix d'un $\mathrm{kg}$ de poisson frais revient à $0,85 \$$ en moyenne (Siegfried, 2018) alors que la présente étude montre que le prix varie d'une part en fonction de la période de l'année, il diminue pendant la période de bonne production $(1,25$ et $1,88 \$)$ et augmente pendant la mauvaise période de production (2 $\$$ et plus). D'autre part, la position géographique qu'occupe le camp de pêche est déterminante ainsi que son accessibilité facile ou non. C'est le cas des camps Port, Muchanga et Mugumba qui sont au centre de Kasenyi, accessibles par route où l'on remarque que le prix d'un kg de poissons est toujours plus élevé par rapport aux autres camps (Tableaux 12 et 13). Le camp Kanana qui se trouve au cœur de
Semliki, dans une zone humide où les aménagements humains sont suspendus sur l'eau et ne sont accessibles que par pirogue, le prix du poisson demeure plus faible que dans tous les autres camps de pêche.

Il se dégage aussi une forte relation entre la quantité du poisson capturée et le revenu par unité d'effort de pêche (Figure 4) et cela varie d'un engin de pêche à l'autre. Cette étude montre que le revenu individuel journalier est plus élevé pour la nasse $(31,68 \pm$ $15 \$$ pendant la bonne capture et $4,4 \pm 2,6 \$$ faible capture) que les autres engins et techniques de pêche (Tableau 14). L'analyse des tableaux 4 et 8 fait ressortir clairement que la nasse utilise 1 ou 2 personnes et une barque non motorisée. On comprend donc qu'elle n'implique pas beaucoup de moyens (dépenses) et toute la capture, bien que faible $(20 \pm 6,9 \mathrm{Kg}$ en moyenne par sortie contre $62,79 \pm 23,24 \mathrm{Kg}$ et $62,74 \pm 21,07 \mathrm{Kg}$ respectivement pour les filets dormant de surface et celui de fond lorsque la capture est bonne), appartient à une ou deux personnes au maximum. La senne de tournante qui emploie entre 8 et 10 personnes en général et bien qu'elle donne des captures plus élevées (183,18 $\pm 49,02 \mathrm{Kg}$ lors des bonnes captures) que la nasse, a un revenu individuel, après retrait des dépenses relatives aux activités de pêche, inférieur à celui de la nasse. Néanmoins la senne procure à son propriétaire (armateur) plus d'intérêt car la capture est divisée entre lui et les ouvriers pêcheurs comme le montre le résultat.

Lelievre (2011) signale que les zones de frayère sont sensibles et beaucoup convoitées par les pêcheurs. La destruction de ces dernières et la pêche des poissons de petite taille conduisent à l'effondrement de stock (Fromentin et Fonteneau, 2001). Il est clair que les zones de frayère du lac Albert sont occupées par les pêcheurs et ces derniers capturent une diversité de poissons à taille différentes en utilisant une diversité des techniques de pêche pour accroitre le rendement bien qu'ils soient conscients de la diminution de la capture par unité de pêche. Bien que les camps de Koga et Nyanza soient considérés comme installés dans la zone de 
frayère par les services étatiques, il y a lieu d'approfondir cette question afin de mettre sur pied un plan de gestion durable des pêcheries au lac Albert et plus particulièrement dans la partie sud-ouest de la RD Congo.

\section{Conclusion}

L'analyse des résultats obtenus à l'issue de cette étude nous permet de conclure que : Les activités de pêche concernent plus les hommes que les femmes qui, elles n'interviennent surtout que dans les activités de transformation et de vente des produits halieutiques. La plupart des pêcheurs ont un niveau d'instruction faible ne dépassant pas le cycle d'orientation ( 2 ans post primaires). Les Alurs, particulièrement sont plus impliqués dans l'activité de pêche que d'autres tribus originaires du territoire d'Irumu. La pêche dans la partie sud-ouest du lac Albert (partie RD Congo), utilise un nombre varié d'engins et techniques de pêche. Certains engins notamment prohibés continuent d'être utilisés de manière illicite. Ce qui nous permet de croire que la pêche illicite est en place et le système de contrôle et de surveillance des activités de pêche doit être appuyé, renforcé et reformé, ce qui semble être le cas récemment (confiscation et brulage des sennes, des filets moustiquaires et des filets monofilaments). La pêche est basée sur les espèces des familles Alestidae, Cichlidae, Bagridae, Mochokidae, Latidae, Cyprinidae, Schilbeidae et Clariidae ayant une bonne valeur marchande, du moins pour les espèces de grande taille. Les filets dormants de surface, de fond et la nasse sont plus sélectifs par rapport aux espèces cibles que la senne tournante qui capture diverses espèces confondues. La capture par Unité d'Effort de Pêche (CPUE) varie en fonction des engins et techniques de pêche d'une part et des périodes de l'année (mois) d'autre part. Malgré ces variations, les revenus des armateurs et des pêcheurs actifs (ouvriers pêcheurs) sont toujours assurés bien qu'ils sont périodiques en utilisant plus d'effort de pêche. Il est nécessaire d'approfondir des études sur l'impact des engins et techniques de pêche afin de ressortir les problèmes relatifs aux différentes pratiques et engins au lac Albert.
Cela permettra d'orienter les actions en faveur de la protection de l'ichtyofaune du lac Albert d'une part et la réglementation de la pêche basée sur des données scientifique d'une part.

\section{CONFLIT D'INTERETS}

Les auteurs déclarent n'avoir aucun conflit d'intérêts.

\section{CONTRIBUTIONS DES AUTEURS}

CK a organisé la collecte des données, analysé et rédigé le manuscrit de l'article ; JM et $\mathrm{JJ}$ ont contribué à la collecte des données ; $\mathrm{AK}, \mathrm{JCM}$ et NM ont guidé les tests et corrigé le manuscrit de l'article.

\section{REMERCIEMENTS}

Ce travail a été réalisé avec le concours des chefs de camp de pêche, les agents du service de l'agriculture basés à Kasenyi, les armateurs et les pêcheurs ouvriers actifs sur le lac Albert. Nous ne cessons de remercier le chef de Kasenyi pour son assistance et son accompagnement pendant notre séjour au lac.

\section{REFERENCES}

Badahoui A, Fiogbe ED, Boko M. 2010. Les causes de la dégradation du lac Ahémé et ses chenaux. Int. J. Biol. Chem. Sci., 4(4): 882-897.

Balole bwami E, Mumbere JC, Matunguru JM, Kujirakwinja D, Shamavu P, Muhindo E, Tchouamo IR, Baudown M, Micha JC. 2018. Production et impact de la pêche dans la partie congolaise du lac Edouard, Tropicultura, 36 (3): 539-552.

Baker S. W. 1869. Le lac Albert: Nouveau Voyage aux Sources du Nil (abrégé d'après la traduction de Gustave Masson par J. Belin-de Launay). Hachette : Paris ; $360 \mathrm{p}$.

Bartley DM, DeGraaf GJ, Valba-Jorgensen J, Marmulla G. 2015. Inland capture fisheries status and data issues, Fisheries Management and Ecology, 22 (1): 71-77.

Bergh MO, Sowman M. 2016. Impact socioéconomiques et environnementaux de la pêche illégale, non déclarée et non réglementaire (IUU) en Afrique, Bureau 
interafricain des ressources animales. Union Africaine, $15 \mathrm{p}$.

Bongeba C, Micha J-C. 2013. Etat de la pêche au Sud du lac Mai-Ndombe. Revue Scientifique et Technique Forêt et Environnement du Bassin du Congo, 1 : 46-55.

Burac M, Medouze C, Joseph P, Gandrille B. 2006. La Caraïbe, Données Environnementales. Edition KARTHALA : Paris ; 453 p.

Chauveau J-P, Jul-Larsen E, Chaboud C. 2000. Les Pêches Piroguières en Afrique de l'Ouest. Edition KARTHALA: Paris; $383 \mathrm{p}$.

CRHOB. 2004. Schéma d'exploitation des fonds marins du plateau continental béninois, Cotonou, $49 \mathrm{p}$.

FAO. 2008. Gestion de l'effort de pêche. Rome, $147 \mathrm{p}$.

FAO, 2010: Directives techniques pour une pêche responsable: les dimensions humaines de l'approche écosystémique des pêches. Rome, 128 p.

FAO. 2018. La situation mondiale de la pêche et de l'aquaculture: Atteindre les objectifs du développement durable. Rome CC BY-NC-SA 3.0 IGO, 254 p.

Fermon Y. 2007. La clé de détermination des familles de poissons du lac Albert, Paris, $20 \mathrm{p}$.

Hounsounou LC. 2011. Importance et place socioéconomique de pêche à la senne de plage dans les moyens d'existence des communautés de pêche au Bénin, Cotonou, $65 \mathrm{p}$.

Hounsounou LC, Sohou Z, Akouchou G. 2013. Pêche à la senne de plage au Benin et durabilité des ressources halieutiques. Journal Recherche Scientifique Universitaire, Togo, Série A, 15(3): 1-13.

Fromenten JM, Fonteneau A. 2001. Fishing effects and life history traits: a cose study comparing tropical versus temperature tunas, Fisheries research, 689-701.

Idowu AA. 2010. Effect of some socioeconomic activities on fish diversity of lagoon systems in Ogun waterside Local Government of Ogun State, Nigeria. Int. J. Biol. Chem. Sci., 4(1): 88-99.
Kraidy AB, Kone N, Berte S, N'zi Konan G, Yao SS, Kouamelan PE. 2014. Pêche et paramètres de reproduction de Pellonula leonensis Boulenger 1916, dans le lac de Taabo (Fleuve Bandama, Côte d'Ivoire): implications pour une exploitation durable du stock. Int. J. Biol. Chem. Sci., 8(1): 75-88.

Laë R, Lévêque CD. 1999. La pêche. In Les Poissons des Eaux Continentales Africaines: Diversité, Ecologie, Utilisation par l'Homme, Lévêque C, Paugy D (eds). IDR : Paris ; 385-424.

Lelievre S. 2011. Identification et caractérisation des frayères hivernales en Manche Orientale et la partie sud de la mer du Nord : Identification des œufs de poissons, cartographie et modélisation des habitats de ponte, Thèse de doctorat, Université de Nantes, 334 p.

Lévêque CD, Paugy D, Teugels G. 2003. Faune des poissons d'eaux douces et saumâtres de l'Afrique de l'Ouest. Paris, OrstomMRAC. Coll. Faune Tropicale, 28(1) : 356-358.

Lévêque CD, Paugy D. 1999b. Caractéristique générale de la faune ichtyologique. In Les Poissons des Eaux Continentales Africaines: Diversité, Ecologie, Utilisation par l'Homme, Lévêque C, Paugy D (eds). IRD : Paris ; 43-53.

Lévêque CD, Paugy D. 1999c. Impact des activités humaines. In Les Poissons des Eaux Continentales Africaines: Diversité, Ecologie, Utilisation par l'Homme, Lévêque C, Paugy D (eds). IRD : Paris ; 365-383.

Luhusu KF, Micha J-C. 2013. Analyses des modes d'exploitation des ressources halieutique du lac Mai-Ndombe en République Démocratique du Congo. Geo-Eco-Trop, 37(2) : 273-284.

Messen JTH. 1951. Monographie de l'Ituri : Histoire, Géographie et Economie. Ministère des Colonies: Royaume de Belgique ; $327 \mathrm{p}$.

Micha J-C, Franck V. 2004. Etude perspective pour la relance du secteur pêche et aquaculture en Côte d'Ivoire, Ministère 
de la Production Animale et des Ressources Halieutiques, Abidjan, 60 p.

Mukabo G, Micha J-C, Habarugira JB, Ntakimazi G, Nshombo, Bizuru VP, Muhirwa BG. 2017. Socio-économie de la pêche artisanale dans les eaux burundaises du lac Tanganyika à Mvugo et Mugaruka. Int. J. Biol. Chem. Sci., 11(1). 247-265.

Paugy D, Lévêque CD. 2017. Impacts of human activities; The inland water fishes of Africa: Diversity, Ecology and Human use. Institut de Recherche pour le Développement (IRD), Royal Museum for Central Africa (RMCA), 459-478.

Siegfried B, Makhtar T. 2018. Analyse de la chaîne de valeur au Sénégal, Rapport, 40 p.

Ssentongo GW. 1984. Projet PPEC exploitation des pêcheries et gestion rationnelle des lacs Edouard et Mobutu/Albert, $24 \mathrm{p}$.

Tah L, Da Costa KS, Kouassi JN, Moreau J. 2009. Effort de pêche et production piscicole au lac Ayamé I (Bassin de la Bia, Côte d'Ivoire) après le départ des pêcheurs Bozos. Agronomie Africaine, 21(1) : 103-115.

Teugels G, Mélanie LJ, Hopkins CD. 2007. Poissons d'eaux douces et saumâtres de Basse Guinée, Ouest de l'Afrique Centrale, Paris. Coll. Faune et Flore 42, Musée Royal de l'Afrique Centrale, Tervuren, IRD et MNHN, 512-530.

Ticheler A. 2000. Conservation de la biodiversité des poissons dans les zones humides de l'Afrique de l'Ouest, Plan d'action Régional 2000, Wetlands International. 